\title{
Non-Hermitian topological end-mode lasing in polariton systems
}

\author{
P. Comaron $\odot,{ }^{1,{ }^{*}}$ V. Shahnazaryan,,${ }^{1,2}$ W. Brzezicki, ${ }^{3}$ T. Hyart, ${ }^{3}$ and M. Matuszewski ${ }^{1}$ \\ ${ }^{1}$ Institute of Physics, Polish Academy of Sciences, Al. Lotników 32/46, 02-668 Warsaw, Poland \\ ${ }^{2}$ ITMO University, St. Petersburg 197101, Russia \\ ${ }^{3}$ International Research Centre MagTop, Institute of Physics, Polish Academy of Sciences, Al. Lotników 32/46, 02-668 Warsaw, Poland
}

(Received 19 December 2019; accepted 21 May 2020; published 5 June 2020)

\begin{abstract}
We predict the existence of non-Hermitian topologically protected end states in a one-dimensional excitonpolariton condensate lattice, where topological transitions are driven by the laser pump pattern. We show that the number of end states can be described by a Chern number and a topological invariant based on the Wilson loop. We find that such transitions arise due to enforced exceptional points which can be predicted directly from the bulk Bloch wave functions. This allows us to establish a new type of bulk-boundary correspondence for non-Hermitian systems and to compute the phase diagram of an open chain analytically. Finally, we demonstrate topological lasing of a single end mode in a realistic model of a microcavity lattice.
\end{abstract}

DOI: 10.1103/PhysRevResearch.2.022051

Systematic analysis of symmetries and topological invariants has revealed a rich variety of topological materials which possess robust boundary states stemming from the topology of the bulk bands [1,2]. The next endeavor is to extend these concepts to non-Hermitian (NH) systems [3-15]. While many interesting properties not present in their Hermitian counterparts were predicted to exist [16-23], NH topological states have been realized experimentally in only a handful of systems [24-33]. The vast majority of the theoretical proposals require violation of reciprocity, which is difficult to implement experimentally in both condensed matter and optical systems. Recently it was demonstrated that topologically protected end modes can arise in lattice systems where non-Hermiticity can be expressed as complex-valued diagonal on-site terms [34-36]. A possible realization consists of a lattice of coupled optical waveguides [30,31] where the nontrivial topology originates from the periodic modulation of the gain-loss balance.

In this Rapid Communication we study $\mathrm{NH}$ physics in a light-matter hybrid quantum quasiparticle system [37] and demonstrate the feasibility of $\mathrm{NH}$ topological end states and end-mode lasing. The nontrivial topology is achieved through spatial modulation of the external incoherent pump intensity in a one-dimensional lattice of homogeneously coupled microcavity exciton-polaritons. These are natural candidate systems for realizing topological phases [38,39], but in existing proposals [40-53] and realizations [28,54-57] topological order arises from the band topology in a Hermitian Hamil-

\footnotetext{
*paolocomaron@gmail.com
}

Published by the American Physical Society under the terms of the Creative Commons Attribution 4.0 International license. Further distribution of this work must maintain attribution to the author $(s)$ and the published article's title, journal citation, and DOI. tonian, so it is not related to the open dissipative $\mathrm{NH}$ nature of the system. We show that the presence of an excitonic component opens up the possibility of realizing a polariton system with competing Hermitian and non-Hermitian effects, so that unique topological phases possessing various numbers of topological end states can be observed. In particular limits of the model the number of end states is described by the Chern number [23] and a topological invariant based on the eigenvalues of the Wilson loop [58], but by continuity the end states exist beyond these special cases. We find that the end modes can disappear or appear at transitions where the energy gap between the bulk bands and the end states closes without closing of the bulk energy gap. Such transitions can occur in open NH systems due to high-order exceptional points [21] leading to the NH skin effect [17-19,22,59]. However, we find that the skin effect is not present in our model because the Hamiltonian satisfies a specific type of $\mathrm{NH}$ time-reversal symmetry [15]. We discover that the transitions occurring in an infinite open chain arise because specific bulk Bloch wave functions are incompatible with the boundary conditions, leading to enforced exceptional points in the spectrum. In this way we establish a new type of bulk-boundary correspondence for NH Hamiltonians, because the changes in the number of end states can be directly predicted from the bulk Bloch wave functions. This powerful new tool allows us to compute the topological phase diagram of an infinite open system analytically.

Moreover, we demonstrate the possibility of lasing of end modes for realistic physical parameters corresponding to a lattice of polariton micropillars. By appropriate choice of the pumping intensity pattern, we achieve a situation where only the selected end state is amplified, which results in topological single-mode lasing.

We consider a one-dimensional lattice of coupled micropillars as depicted in Fig. 1(a). Each micropillar contains a quantum well and is assumed to host a tightly bound excitonpolariton mode [60]. We start with a system of discrete 

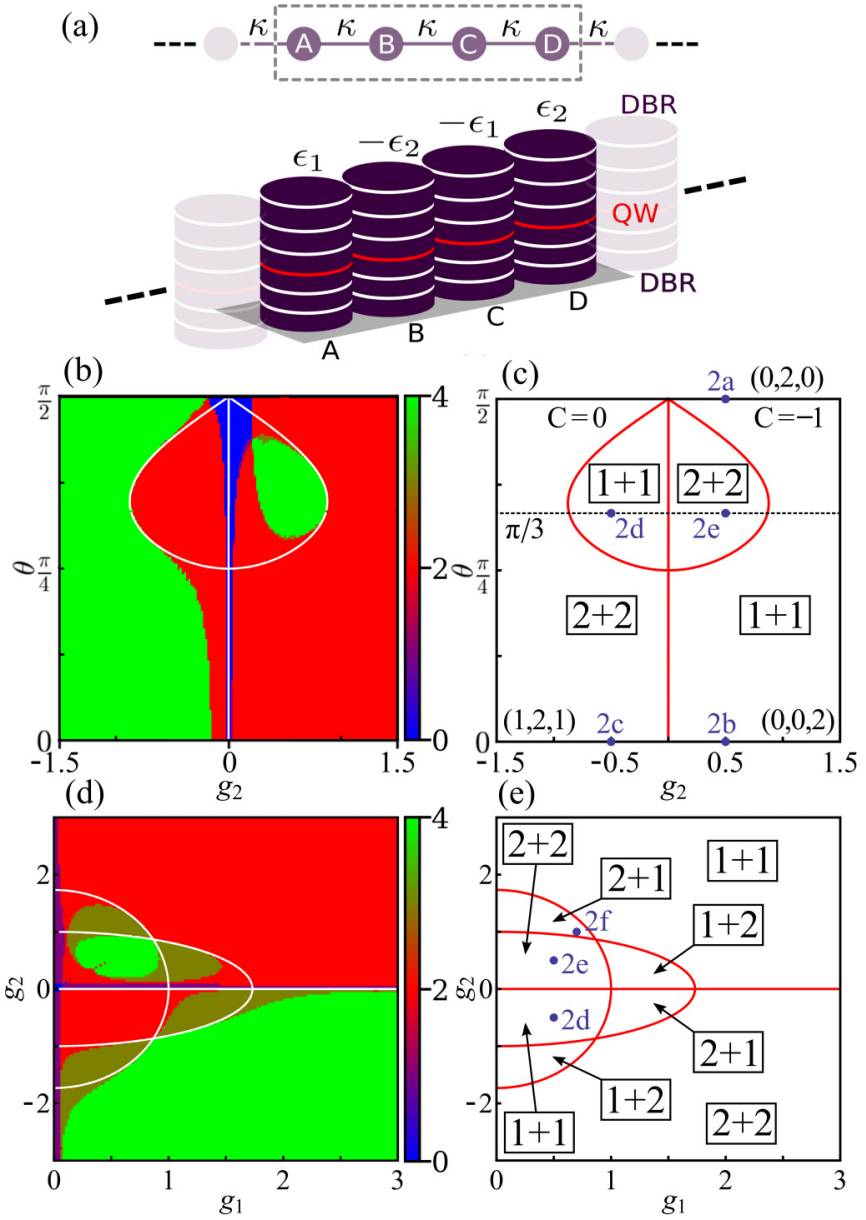

FIG. 1. (a) Non-Hermitian four-site unit-cell lattice of microcavity pillars with embedded quantum wells. (b) Topological phase diagram of the symmetric system $g_{1}=\left|g_{2}\right|$ obtained by counting the end states as a function of the pumping modulation amplitudes $g_{1,2}$ and non-Hermiticity parameter $\theta$. The length of the chain in the numerical calculation is $L=4 \times 100$. (c) Topological phase diagram for an infinite open chain with $g_{1}=\left|g_{2}\right|$ obtained by combining the information from the Chern number $C$, Wilson loop invariant, and enforced exceptional points. The phases with $M_{L}\left(M_{R}\right)$ localized modes in the left (right) end of the chain are denoted $\left[M_{L}+M_{R}\right]$. In the special cases where the bulk bands and the end states can be ordered based on the real part of the energies we use the notation $\left(m_{1}, m_{2}, m_{3}\right)$, where $m_{i}$ is the number of end states in the $i$ th energy gap. (d, e) Numerically and analytically computed phase diagrams as a function of $g_{1}$ and $g_{2}$ for $\theta=\pi / 3$. The small disagreement between the phase diagrams arises due to the finite-size effects. All energies are in units of $\kappa$.

mean-field Gross-Pitaevskii equations [61,62],

$$
\begin{aligned}
i \hbar \dot{\psi}_{n} & =-\kappa \sum_{\langle n n\rangle} \psi_{m}+\left[g_{c}\left|\psi_{n}\right|^{2}+g_{R} n_{n}^{R}+i \hbar \frac{R n_{n}^{R}-\gamma_{c}}{2}\right] \psi_{n}, \\
\dot{n}_{n}^{R} & =P_{n}-\left(\gamma_{R}+R\left|\psi_{n}\right|^{2}\right) n_{n}^{R},
\end{aligned}
$$

where $\psi_{n}(t)$ is the condensate amplitude in the $n$th lattice cell, the $\langle n n\rangle$ sum runs over nearest neighbors, $n_{n}^{R}(t)$ is the density of the exciton reservoir in the $n$th cell, $P_{n}$ is the external nonresonant pumping rate, $\gamma_{c}$ and $\gamma_{R}$ are the decay rates of the condensate and the reservoir, respectively, $g_{c}$ and $g_{R}$ are the corresponding interaction constants, and $R$ is the rate of scattering from the reservoir to the condensate. We assume that the polariton interactions within the condensate represented by the term $g_{c}\left|\psi_{n}\right|^{2}$ are negligible in comparison with the reservoir-condensate interaction $g_{R} n_{n}^{R}$, which is a good approximation in most experiments where nonresonant pumping is used.

While the coupling coefficients $\kappa$ are assumed to be the same for each pair of neighboring micropillars, the external pumping $P_{n}$ is modulated spatially with the four-site periodicity. Therefore, the pumping gives rise to a complex on-site potential [Fig. 1(a)]. Within the adiabatic approximation [63,64], this leads to an effective NH tight-binding Hamiltonian:

$$
\hat{H}_{k}\left(g_{1}, g_{2}\right)=\left(\begin{array}{cccc}
g_{1} e^{i \theta} & \kappa & 0 & \kappa e^{-i k} \\
\kappa & -g_{2} e^{i \theta} & \kappa & 0 \\
0 & \kappa & -g_{1} e^{i \theta} & \kappa \\
\kappa e^{i k} & 0 & \kappa & g_{2} e^{i \theta}
\end{array}\right) .
$$

Here $k$ is the Bloch wave number in units of inverse intercell distance, and $\epsilon_{1,2}=g_{1,2} e^{i \theta}$ are the complex on-site potentials, which are given by $\epsilon_{n}=\left(n_{n}^{R}-\gamma_{c} / R\right)\left(g_{R}+i \hbar R / 2\right)$. The real part of $\epsilon_{1,2}$ stands for the interaction-induced on-site potential, whereas the imaginary part corresponds to the balance between gain and loss. In the linear approximation of negligibly small $\left|\psi_{n}\right|^{2}$, which we assume in most of this work, the reservoir occupation $n_{n}^{R}$ in the above equation is defined by the local pumping rate and takes the form $n_{n}^{R}=n_{n 0}^{R}=P_{n} / \gamma_{R}$, and we have chosen the pump rates $P_{n}$ so that the on-site potentials at every second lattice site are related to each other. The real amplitudes $g_{n}$ can be positive or negative depending on the local pumping intensity [63]. For realistic repulsive interactions between polaritons one has $0<\theta<\pi / 2$. When complex on-site potentials are absent $\left(\epsilon_{1,2}=0\right)$, the lattice is trivial since all the coupling coefficients $\kappa$ are equal. In typical polariton micropillar lattices $\kappa \sim 0.1 \mathrm{meV}$.

We solved Hamiltonian (2) numerically with open boundary conditions and calculated the number of end states as a function of various parameters of the model [63]. The results are shown in Figs. 1 and 2. We denote the phases with $\left[M_{L}+M_{R}\right]$, where $M_{L}\left(M_{R}\right)$ is the number of modes localized in the left (right) end. In the special cases where we can order the bulk bands and the end states based on the real part of the energies we also use the notation $\left(m_{1}, m_{2}, m_{3}\right)$, where $m_{i}$ is the number of end states in the energy gap between the $i$ th and the $i+1$ th bands.

In the extreme $\mathrm{NH}$ limit $\theta=\pi / 2$ the system obeys $\mathrm{NH}$ chiral symmetry $\mathcal{S} \hat{H}_{k}\left(g_{1}, g_{2}\right) \mathcal{S}=-\hat{H}_{k}^{\dagger}\left(g_{1}, g_{2}\right)$ with $\mathcal{S}=\mathbb{1} \otimes$ $\sigma_{z}$. In this case the number of end states with zero real part of the energy is described by the Chern number $C[23,63]$. For $g_{2}>0$ and $g_{1}=\left|g_{2}\right|$ we obtain $C=-1$, which means that there should be one state with zero real part of the energy at each end of the chain in agreement with our numerical calculations [Figs. 1(c) and 2(a)]. Thus, this limiting case of the model belongs to the $[1+1]$ phase, with the number of end states in each energy gap given by $(0,2,0)$. In this limit the system exhibits topological states solely due to the non-Hermiticity [34]. 

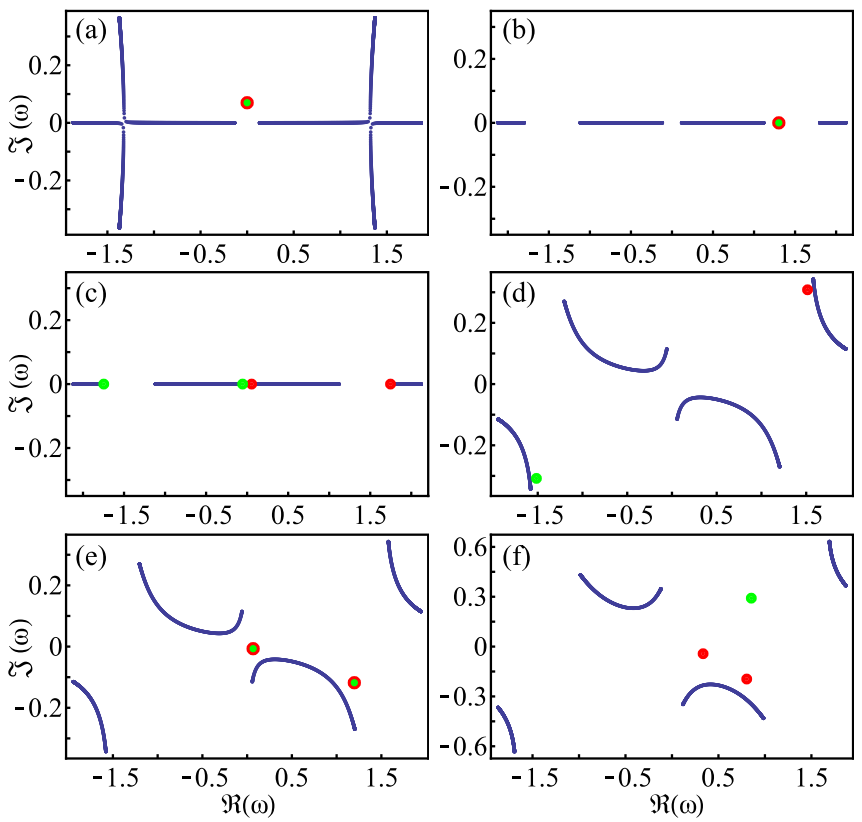

FIG. 2. Complex energy spectra of an open chain with $L=1000$ for the topological phases shown in Figs. 1(c) and 1(e). Red (green) dots denote end states at the left (right) end of the chain. The parameters are (a) $g_{1}=g_{2}=0.5, \theta=\pi / 2$; (b) $g_{1}=g_{2}=0.5, \theta=$ 0 ; (c) $-g_{1}=g_{2}=-0.5, \theta=0$; (d) $-g_{1}=g_{2}=-0.5, \theta=\pi / 3$; (e) $g_{1}=g_{2}=0.5, \theta=\pi / 3$; (f) $g_{1}=0.7, g_{2}=1.0, \theta=\pi / 3$. All energies are in units of $\kappa$.

If $\theta=0, g_{1}=\left|g_{2}\right|$, and $g_{2}>0$, the system is Hermitian and obeys an inversion symmetry $\mathcal{I} \hat{H}_{k}(g, g) \mathcal{I}^{-1}=\hat{H}_{-k}(g, g)$, where $\mathcal{I}=\sigma_{x} \otimes \sigma_{x}$. Therefore, a topological invariant can be defined based on a Wilson loop invariant or quantized Zak phases $[58,63]$. We find that both approaches yield identical predictions [63]. Namely, we find that the first two energy gaps are trivial, whereas the third energy gap is nontrivial [Fig. 2(b)]. Thus, the number of end states in each energy gap is given by $(0,0,2)$ [Fig. $1(\mathrm{c})]$.

If $\theta=0, g_{1}=\left|g_{2}\right|$, and $g_{2}<0$, the bulk Hamiltonian obeys inversion symmetry but the unit cell is not compatible with the inversion symmetry. This obscures the bulk-boundary correspondence but nonrigorous reasoning based on Wilson loop invariants indicates that the model belongs to the $[2+2]$ phase with the number of end states $(1,2,1)$ [63], in agreement with numerical calculations [Fig. 2(c)].

Although in a realistic description of exciton-polaritons one has $0<\theta<\pi / 2$, the limits considered above are useful because end states can disappear or appear only when their energy approaches that of bulk bands. Therefore, we obtain the overall phase diagram shown in Figs. 1(b) and 1(c). There is an energy gap closing between bands if $g_{1}=g_{2}=0$, so this phase transition is also easy to understand.

We find that if $\pi / 4<\theta<\pi / 2$, there exist additional transitions where the end modes disappear or appear without closing of the bulk gap [Figs. 1(b) and 1(c)]. Such transitions are known to occur due to the NH skin effect [17-19,22], but the skin effect is absent in our model because of NH timereversal symmetry, $\hat{H}_{-k}^{*}\left(g_{1}, g_{2}\right)=\hat{H}_{k}^{\dagger}\left(g_{1}, g_{2}\right)$ [15]. Instead, we note that the eigenstates of the continuum bands in an infinite open chain can be constructed from standing waves of bulk Bloch wave functions. This construction follows the one suggested in Ref. [22], but we discover that if the first or last component of a bulk Bloch wave function vanishes, it is impossible to satisfy the boundary conditions. Therefore, states should disappear from continuum bands at parameter values where such incompatibility occurs. A connection between boundary conditions and exceptional points was also pointed out in Ref. [65] but the relation to the bulk wave functions (i.e., bulk-boundary correspondence) was not discovered in this earlier work. This can happen in NH systems with the help of exceptional points which transform into new end states. In the following we demonstrate that with the help of this reasoning we can compute the topological phase diagram of an infinite open system analytically.

To find the enforced exceptional points in an infinite open chain we need to calculate when the product $Q_{k}$ of the first and the last components of all eigenvectors vanishes. In the case $g_{1}=\left|g_{2}\right|$ we find by a straightforward calculation that

$$
Q_{k} \propto f_{k} f_{-k}, \quad f_{k}=4 g_{2}^{2} e^{i(k+2 \theta)}+\kappa^{2}\left(1+e^{i k}-e^{2 i k}-e^{3 i k}\right) .
$$

Therefore, $Q_{k}$ vanishes along the contour

$$
g_{2}=\kappa \frac{\sin k}{\sqrt{2\left|\sin \frac{k}{2}\right|}}, \quad \theta=\frac{\pi+|k|}{4}, \quad k \in[-\pi, \pi) .
$$

The vanishing $Q_{k}$ leads to the appearance of two exceptional points because of inversion (chiral-inversion) symmetry if $g_{2}>0\left(g_{2}<0\right)$. Therefore, the number of end states changes by 2 at these transition lines. By computing the end-state spectrum for $g_{2}>0\left(g_{2}<0\right)$ we indeed find that the $[1+1]$ $([2+2])$ phase transforms into the $[2+2]([1+1])$ phase when $g_{2}$ and $\theta$ are tuned across the transition line defined by Eq. (3) [Figs. 1(b) and 1(c) and Figs. 2(d) and 2(e)]. In addition to the prediction of the phase boundaries, we can predict the momentum and the band where the exceptional point leads to the appearance or disappearance of end states. We checked that numerical calculations are in agreement with these analytical considerations (see [63]).

Similarly we can find the zeros of $Q_{k}$ in the $g_{1}-g_{2}$ plane for fixed $\theta$. The explicit expression for $Q_{k}$ is more complicated but we arrive at the simple result that $Q_{k}$ vanishes along the four elliptic contours

$$
g_{1}= \pm \kappa \cos \frac{k}{2}, \quad g_{2}= \pm \kappa \tan \theta \sin \frac{k}{2}, \quad k \in[-\pi, \pi)
$$

and

$$
g_{1}= \pm \kappa \tan \theta \sin \frac{k}{2}, \quad g_{2}=\mp \kappa \cos \frac{k}{2}, \quad k \in[-\pi, \pi) .
$$

In the general case $g_{1} \neq\left|g_{2}\right|$ the vanishing of $Q_{k}$ leads to a single exceptional point so that the number of end states changes by 1 at these transition lines. Thus, we obtain the exotic phases $[1+2]$ and $[2+1]$, with different numbers of topological states at the two ends of the chain. Our numerical calculations are in agreement with these analytic considerations [Figs. 1(d)-1(f)]. Thus, our results demonstrate that the transitions occurring in an infinite open chain can be predicted from the Bloch wave functions, establishing a new type of bulk-boundary correspondence for non-Hermitian systems. 


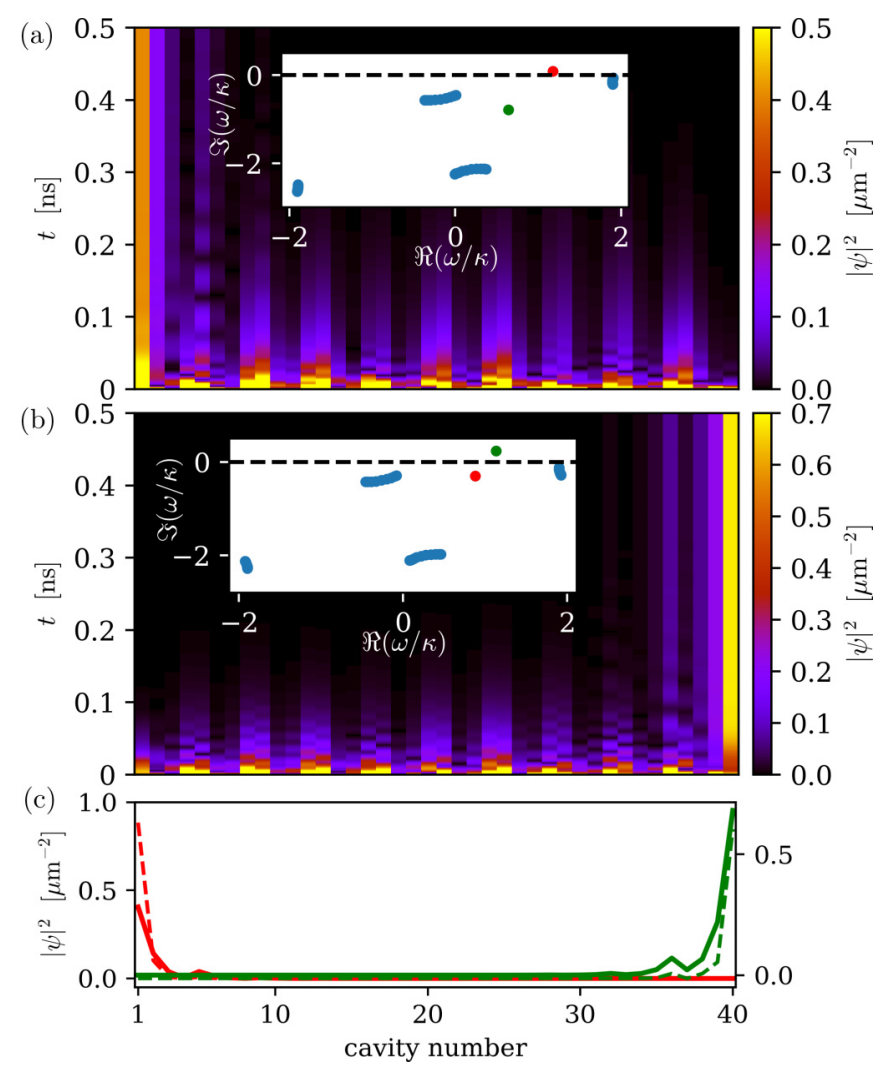

FIG. 3. The emergence of end-mode lasing in the nonlinear model, (4). The color scale shows the evolution of the field density in each cavity for the physical parameters: (a) $g_{1}=0.2 \mathrm{meV}$, $g_{2}=0.1 \mathrm{meV}$, and $\hbar \gamma=0.135 \mathrm{meV}$; and (b) $g_{1}=0.14 \mathrm{meV}, g_{2}=$ $0.14 \mathrm{meV}, \hbar \gamma=0.12 \mathrm{meV}$. In the rightmost pillar we apply an extra pump so that there the value of $g_{2}$ is modified to $0.196 \mathrm{meV}$. Insets in (a) and (b): The corresponding spectra of an open chain in the linear limit as in Fig. 2. (c) Steady-state density profiles after a long evolution time for the cases shown in (a) as the solid red line and (b) as the solid green line are compared to the corresponding end-state densities (dashed lines) calculated from the eigenvalues in the linear limit. Other parameters are $\kappa=0.1 \mathrm{meV}$, $\gamma_{c}=1.52 \mathrm{ps}^{-1}, \gamma_{R}=0.075 \mathrm{ps}^{-1}, R=6.9 \times 10^{-3} \mathrm{ps}^{-1} \mu \mathrm{m}^{2}, g_{R}=$

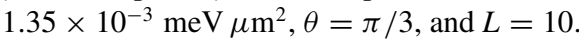

We now consider the possibility of lasing of these end states. We expect that single-mode lasing can be realized if all modes decay in time except a selected end mode, which is amplified by the medium. This requires an adjustment of the spectra presented in Fig. 2. As demonstrated in the Supplementary Information [63], the following set of coupled equations describes the evolution in the weakly nonlinear regime,

$$
i \hbar \dot{\psi}_{n}=-\kappa \sum_{\langle n n\rangle} \psi_{m}+\epsilon_{n} \psi_{n}-\Gamma_{n}(1+i \tan \theta)\left|\psi_{n}\right|^{2} \psi_{n},
$$

where now we have included the additional loss $\gamma$ in each node, i.e., $\epsilon_{\mathrm{A}}=\epsilon_{1}-i \hbar \gamma, \epsilon_{\mathrm{B}}=-\epsilon_{2}-i \hbar \gamma, \epsilon_{\mathrm{C}}=-\epsilon_{1}-i \hbar \gamma$, and $\epsilon_{\mathrm{D}}=\epsilon_{2}-i \hbar \gamma$. This results in a shift of the imaginary part of the excitation spectrum by $-\hbar \gamma$. With a careful choice of $\gamma$, we can stabilize all the eigenstates except one. Physically, it corresponds to a homogeneous reduction of pumping across the lattice. The reduction of pumping affects also the real part of the potential, but this can be eliminated by a rotating frame for the condensate amplitudes [63]. The parameter $\Gamma_{n}=P_{n} R g_{R} / \gamma_{R}^{2}$ describes the nonlinear interactions with the reservoir.

In Fig. 3 we show examples of the evolution of the intensity distributions obtained by solving Eq. (4) in an $L=4 \times 10$ unit-cell configuration with a random initial amplitude. After initial evolution, the polariton density saturates at a steadystate distribution, which approximately corresponds to the end mode in the linear spectrum [see Fig. 3(c)].

In the symmetric case with $g_{1}=\left|g_{2}\right|$ end states are always below bulk states in the imaginary part of the spectrum. To establish single-end-mode lasing, one has to apply an additional pump to one of the end pillars [28]. This leads to an increase in the imaginary part of the corresponding end state, relative to all other states. The resulting imaginary part of the spectrum is shown in the inset in Fig. 3(b). Here the values of the on-site effective potentials $g= \pm 0.14 \mathrm{meV}$ can be created with external pumps $P_{+} \approx 16.45 \mu \mathrm{m}^{-2} \mathrm{ps}^{-1}$ and $P_{-} \approx 8.45 \mu \mathrm{m}^{-2} \mathrm{ps}^{-1}$. In addition, we apply an extra pump to the rightmost pillar with $\delta P \approx 6.58 \mu \mathrm{m}^{-2} \mathrm{ps}^{-1}$.

In conclusion, we predicted a new type of bulk-boundary correspondence for non-Hermitian systems, based on enforced exceptional points. These topological modes can be realized in realistic polariton micropillar lattices. Our general model applies to any non-Hermitian system where competition between gain, loss, and interactions exists, such as in cold-atom systems.

We acknowledge support from National Science Center, Poland, Grants No. 2016/22/E/ST3/00045 and No. 2017/25/Z/ST3/03032 under the QuantERA program. V.S. acknowledges support from Mega-grant No. 14.Y26.31.0015 of the Ministry of Education and Science of Russian Federation. The research was partially supported by the Foundation for Polish Science through the IRA Programme cofinanced by the E.U. within Small Growth Operational Programme.
[1] M. Z. Hasan and C. L. Kane, Rev. Mod. Phys. 82, 3045 (2010).

[2] C.-K. Chiu, J. C. Y. Teo, A. P. Schnyder, and S. Ryu, Rev. Mod. Phys. 88, 035005 (2016).

[3] K. Esaki, M. Sato, K. Hasebe, and M. Kohmoto, Phys. Rev. B 84, 205128 (2011).

[4] H. Schomerus, Opt. Lett. 38, 1912 (2013).

[5] T. E. Lee, Phys. Rev. Lett. 116, 133903 (2016).

[6] H. Menke and M. M. Hirschmann, Phys. Rev. B 95, 174506 (2017).
[7] S. Ke, B. Wang, H. Long, K. Wang, and P. Lu, Opt. Express 25, 11132 (2017)

[8] Y. Xu, S.-T. Wang, and L.-M. Duan, Phys. Rev. Lett. 118, 045701 (2017).

[9] X. Ni, D. Smirnova, A. Poddubny, D. Leykam, Y. Chong, and A. B. Khanikaev, Phys. Rev. B 98, 165129 (2018).

[10] L. Zhou, Q.-h. Wang, H. Wang, and J. Gong, Phys. Rev. A 98, 022129 (2018). 
[11] C. Li, X. Z. Zhang, G. Zhang, and Z. Song, Phys. Rev. B 97, 115436 (2018).

[12] V. M. Martinez Alvarez, J. E. Barrios Vargas, M. Berdakin, and L. E. F. Foa Torres, Eur. Phys. J.: Spec. Top. 227, 1295 (2018).

[13] Z. Gong, Y. Ashida, K. Kawabata, K. Takasan, S. Higashikawa, and M. Ueda, Phys. Rev. X 8, 031079 (2018).

[14] H. Zhou and J. Y. Lee, Phys. Rev. B 99, 235112 (2019).

[15] K. Kawabata, K. Shiozaki, M. Ueda, and M. Sato, Phys. Rev. X 9, 041015 (2019).

[16] D. Leykam, K. Y. Bliokh, C. Huang, Y. D. Chong, and F. Nori, Phys. Rev. Lett. 118, 040401 (2017).

[17] S. Yao and Z. Wang, Phys. Rev. Lett. 121, 086803 (2018).

[18] S. Yao, F. Song, and Z. Wang, Phys. Rev. Lett. 121, 136802 (2018).

[19] F. K. Kunst, E. Edvardsson, J. C. Budich, and E. J. Bergholtz, Phys. Rev. Lett. 121, 026808 (2018).

[20] H. Shen, B. Zhen, and L. Fu, Phys. Rev. Lett. 120, 146402 (2018).

[21] V. M. Martinez Alvarez, J. E. Barrios Vargas, and L. E. F. Foa Torres, Phys. Rev. B 97, 121401(R) (2018).

[22] K. Yokomizo and S. Murakami, Phys. Rev. Lett. 123, 066404 (2019).

[23] W. Brzezicki and T. Hyart, Phys. Rev. B 100, 161105(R) (2019).

[24] J. M. Zeuner, M. C. Rechtsman, Y. Plotnik, Y. Lumer, S. Nolte, M. S. Rudner, M. Segev, and A. Szameit, Phys. Rev. Lett. 115, 040402 (2015).

[25] X. Zhan, L. Xiao, Z. Bian, K. Wang, X. Qiu, B. C. Sanders, W. Yi, and P. Xue, Phys. Rev. Lett. 119, 130501 (2017).

[26] L. Xiao, X. Zhan, Z. Bian, K. Wang, X. Zhang, X. Wang, J. Li, K. Mochizuki, D. Kim, N. Kawakami et al., Nat. Phys. 13, 1117 (2017).

[27] S. Weimann, M. Kremer, Y. Plotnik, Y. Lumer, S. Nolte, K. G. Makris, M. Segev, M. C. Rechtsman, and A. Szameit, Nat. Mater. 16, 433 (2017).

[28] P. St-Jean, V. Goblot, E. Galopin, A. Lemaître, T. Ozawa, L. Le Gratiet, I. Sagnes, J. Bloch, and A. Amo, Nat. Photon. 11, 651 (2017).

[29] B. Bahari, A. Ndao, F. Vallini, A. El Amili, Y. Fainman, and B. Kanté, Science 358, 636 (2017).

[30] M. Parto, S. Wittek, H. Hodaei, G. Harari, M. A. Bandres, J. Ren, M. C. Rechtsman, M. Segev, D. N. Christodoulides, and M. Khajavikhan, Phys. Rev. Lett. 120, 113901 (2018).

[31] M. A. Bandres, S. Wittek, G. Harari, M. Parto, J. Ren, M. Segev, D. N. Christodoulides, and M. Khajavikhan, Science 359 (2018).

[32] H. Zhao, P. Miao, M. H. Teimourpour, S. Malzard, R. ElGanainy, H. Schomerus, and L. Feng, Nat. Commun. 9, 981 (2018).

[33] H. Zhou, C. Peng, Y. Yoon, C. W. Hsu, K. A. Nelson, L. Fu, J. D. Joannopoulos, M. Soljačić, and B. Zhen, Science 359, 1009 (2018).

[34] K. Takata and M. Notomi, Phys. Rev. Lett. 121, 213902 (2018).

[35] S. Lieu, Phys. Rev. B 97, 045106 (2018).

[36] T. Yoshida and Y. Hatsugai, Phys. Rev. B 100, 054109 (2019).

[37] I. Carusotto and C. Ciuti, Rev. Mod. Phys. 85, 299 (2013).

[38] T. Karzig, C.-E. Bardyn, N. H. Lindner, and G. Refael, Phys. Rev. X 5, 031001 (2015.

[39] A. V. Nalitov, G. Malpuech, H. Terças, and D. D. Solnyshkov, Phys. Rev. Lett. 114, 026803 (2015).
[40] C.-E. Bardyn, T. Karzig, G. Refael, and T. C. H. Liew, Phys. Rev. B 91, 161413(R) (2015).

[41] O. Bleu, D. D. Solnyshkov, and G. Malpuech, Phys. Rev. B 93, 085438 (2016).

[42] R. Banerjee, T. C. H. Liew, and O. Kyriienko, Phys. Rev. B 98, 075412 (2018).

[43] A. Janot, B. Rosenow, and G. Refael, Phys. Rev. B 93, 161111(R) (2016).

[44] D. D. Solnyshkov, O. Bleu, B. Teklu, and G. Malpuech, Phys. Rev. Lett. 118, 023901 (2017).

[45] M. Sun, D. Ko, D. Leykam, V. M. Kovalev, and I. G. Savenko, Phys. Rev. Applied 12, 064028 (2019).

[46] A. V. Nalitov, D. D. Solnyshkov, and G. Malpuech, Phys. Rev. Lett. 114, 116401 (2015).

[47] R. Ge, W. Broer, and T. C. H. Liew, Phys. Rev. B 97, 195305 (2018).

[48] V. K. Kozin, I. A. Shelykh, A. V. Nalitov, and I. V. Iorsh, Phys. Rev. B 98, 125115 (2018).

[49] Y. V. Kartashov and D. V. Skryabin, Phys. Rev. Lett. 122, 083902 (2019).

[50] Y. V. Kartashov and D. V. Skryabin, Phys. Rev. Lett. 119, 253904 (2017).

[51] C. A. Downing, T. J. Sturges, G. Weick, M. Stobińska, and L. Martín-Moreno, Phys. Rev. Lett. 123, 217401 (2019).

[52] C.-E. Bardyn, T. Karzig, G. Refael, and T. C. H. Liew, Phys. Rev. B 93, 020502(R) (2016).

[53] H. Sigurdsson, G. Li, and T. C. H. Liew, Phys. Rev. B 96, 115453 (2017).

[54] F. Baboux, E. Levy, A. Lemaître, C. Gómez, E. Galopin, L. Le Gratiet, I. Sagnes, A. Amo, J. Bloch, and E. Akkermans, Phys. Rev. B 95, 161114(R) (2017).

[55] M. Milićević, T. Ozawa, G. Montambaux, I. Carusotto, E. Galopin, A. Lemaître, L. Le Gratiet, I. Sagnes, J. Bloch, and A. Amo, Phys. Rev. Lett. 118, 107403 (2017).

[56] S. Klembt, T. Harder, O. Egorov, K. Winkler, R. Ge, M. Bandres, M. Emmerling, L. Worschech, T. Liew, M. Segev et al., Nature 562, 552 (2018).

[57] C. E. Whittaker, E. Cancellieri, P. M. Walker, D. R. Gulevich, H. Schomerus, D. Vaitiekus, B. Royall, D. M. Whittaker, E. Clarke, I. V. Iorsh et al., Phys. Rev. Lett. 120, 097401 (2018).

[58] A. Alexandradinata, X. Dai, and B. A. Bernevig, Phys. Rev. B 89, 155114 (2014).

[59] C. H. Lee and R. Thomale, Phys. Rev. B 99, 201103(R) (2019).

[60] A. Kavokin, J. J. Baumberg, G. Malpuech, and F. P. Laussy, Microcavities (Oxford University Press, New York, 2017).

[61] M. Wouters and I. Carusotto, Phys. Rev. Lett. 99, 140402 (2007).

[62] P. Stepnicki and M. Matuszewski, Phys. Rev. A 88, 033626 (2013).

[63] See Supplemental Material at http://link.aps.org/supplemental/ 10.1103/PhysRevResearch.2.022051 for a discussion of the polariton model, numerical procedure implemented for end-mode detection, enforced exceptional points and end states, Chern number, Wilson loop invariant, Zak phases, point and line gap closing, dependence of the number of end modes on the Wilson loop invariant in the Hermitian case, and global Berry phase.

[64] N. Bobrovska and M. Matuszewski, Phys. Rev. B 92, 035311 (2015).

[65] L. E. F. F. Torres, J. Phys.: Mater. 3, 014002 (2019). 